\title{
First report of cavitary pneumonia due to community-acquired Acinetobacter pittii, study of virulence and overview of pathogenesis and treatment
}

\author{
Romaric Larcher ${ }^{1 *}$ D, Alix Pantel $^{2}$, Erik Arnaud ${ }^{1}$, Albert Sotto ${ }^{3}$ and Jean-Philippe Lavigne ${ }^{2}$
}

\begin{abstract}
Background: Acinetobacter pittii is a nosocomial pathogen rarely involved in community-acquired infections. We report for the first time that $A$. pittii can be responsible for cavitary community-acquired pneumonia and study its virulence, and discuss its pathogenesis and treatment options.

Case presentation: A 45-year-old woman with a history of smoking and systemic lupus was admitted to Nimes University Hospital (France) with coughing and sputum lasting for three weeks. Thoracic CT scanner showed cavitary pneumonia. Broncho-alveolar lavage cultures found community-acquired Acinetobacter calcoaceticusbaumannii complex. The clinical outcome was favourable after twenty-one days of antimicrobial treatment by piperacillin/tazobactam and amikacin then cefepime. Multilocus sequence typing (MLST) analyses identified an A. pittii ST249. Despite the atypical clinical presentation with an unexpected partial destruction of lung parenchyma, we found very low virulence potential of the $A$. pittii strain with nematode killing assays and biofilm formation test. The median time required to kill 50\% of the nematodes was $7 \pm 0.3$ days for $A$. pittii ST249, $7 \pm 0.2$ days for $A$. baumanii NAB ST2 and $8 \pm 0.2$ days for $E$. coli OP50, $(p>0,05)$. A. pittii ST249 showed significantly slower biofilm formation than A. baumanii NAB ST2: $\mathrm{BFI}=8.83 \pm 0.59$ vs $3.93 \pm 0.27$ at $2 \mathrm{~h}(p<0.0001), \mathrm{BFI}=6.3 \pm 0.17$ vs 1 . $87 \pm 0.12$ at $3 \mathrm{~h}(p<0.0001)$ and $\mathrm{BFI}=3.67 \pm 0.41$ vs $1.7 \pm 0.06$ after $4 \mathrm{~h}$ of incubation $(p<0.01)$.
\end{abstract}

Conclusions: Community-acquired A. pittii should be considered as possible cause of sub-acute cavitary pneumonia particularly in a smoking and/or immunocompromised patient despite its low virulence potential.

Keywords: Community-acquired pneumonia, Cavitary pneumonia, Cavitation, Acinetobacter pittii, Acinetobacter calcoaceticus-baumannii complex, Virulence, Biofilm

\section{Background}

The classic underlying causes of lung abscess or cavitary lung lesions are anaerobes, Streptococcus pneumonia, Streptococcus milleri group, Staphylococcus aureus and Klebsiella pneumoniae. More rarely Pseudomonas aeruginosa and other aerobic Gram-negative bacilli, Nocardia spp., Aspergillus spp. and Cryptococcus spp. could be identified [1]. To our knowledge, Acinetobacter pittii, formerly known as Acinetobacter genomospecies 3, has not been

\footnotetext{
* Correspondence: romaric.larcher@gmail.com

${ }^{1}$ Department of Internal Medicine, Caremeau University Hospital, 29 place du Professeur Debre, Nimes, France

Full list of author information is available at the end of the article
}

described as a cause of cavitary pneumonia before and it appears to be an unusual cause of community-acquired infections [1-3]. However, it is increasingly described as a cause of hospital-acquired infection particularly in intensive care unit setting [4]. Here, we report the first case of cavitary community-acquired pneumonia due to A. pittii in a patient with a systemic lupus and study the virulence profile of $A$. pittii.

\section{Case presentation}

A 45-year-old woman, was admitted to Nimes University Hospital (France) for a cough and sputum lasting for three weeks with an unfavourable outcome despite 
prescription of antibiotics (cefixime then pristinamycin). She had a medical history of smoking and systemic lupus diagnosed in 1991 with moderately reduced glomerular filtration rate $\left(57 \mathrm{ml} / \mathrm{min} / 1.73 \mathrm{~m}^{2}\right)$, stroke and right nephrectomy in 2011. Her usual treatment included hydroxychloroquine, clopidogrel and atorvastatine.

At admission, she presented dyspnoea, crackles of the right lung field and normal temperature. The white blood cell count was $19 \times 10^{3}$ cells $/ \mu \mathrm{L}$ and $\mathrm{C}$ reactive protein was high at $144 \mathrm{mg} / \mathrm{L}$. Chest X-ray showed a large pulmonary cavity of the right lower lobe (Fig. 1) and computed tomography (CT-scan) showed four large cavities and nodules of the right lower lobe (Figs. 2 and 3). Blood cultures remained negative. A fibroscopy with broncho-alveolar lavage (BAL) showed local inflammation with sputum. Piperacillin/tazobactam plus amikacin was administered as empirical treatment. The BAL culture was positive with more than $10^{4} \mathrm{CFU} / \mathrm{mL}$ of Gram negative bacilli.

MALDI-TOF (Matrix Assisted Laser Desorption Ionisation/Time Of Flight) analysis (Vitek MS, bioMérieux) identified A. calcoaceticus-baumannii complex (99\%). Antimicrobial susceptibility was tested by disc diffusion in accordance with EUCAST (European Committee on Antimicrobial Susceptibility Testing) recommendations (version 5.0, 2015; http://www.eucast.org). The isolate was susceptible to ticarcillin, piperacillin, third and fourth generation cephalosporins, imipenem, ciprofloxacin and amikacin. Cultures for mycobacteria, Nocardia spp., Aspergillus spp. and Cryptococcus spp. were negative. Bronchial biopsy was normal.

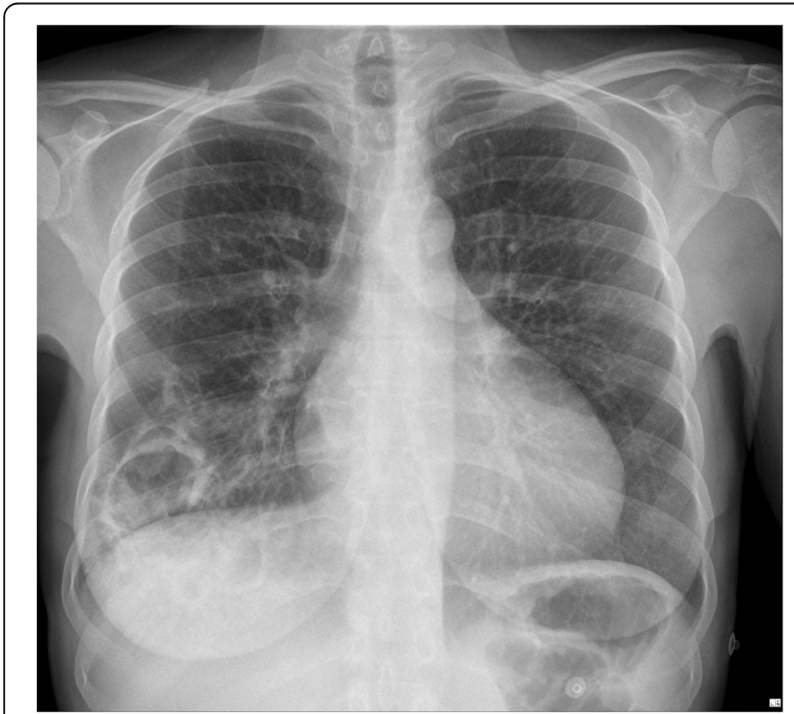

Fig. 1 Chest $X$-ray showing a community-acquired pneumonia due to $A$. pittii with a large pulmonary cavity of the right lower lobe, at hospital admission

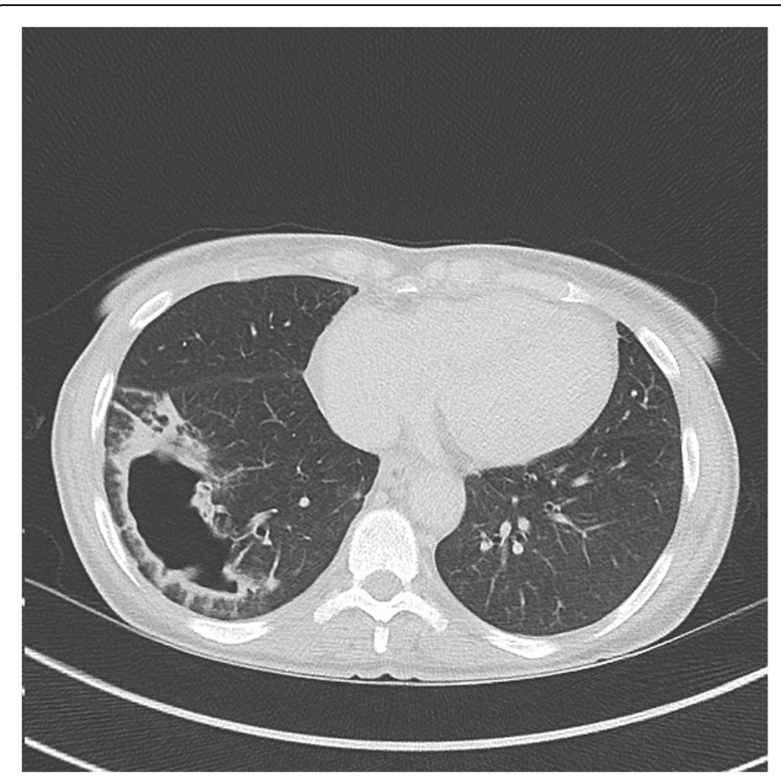

Fig. 2 Thoracic CT scan showing a cavitary community-acquired pneumonia due to $A$. pittii with the largest cavitation of the right lower lobe, three days after admission

Cavitary community-acquired pneumonia due to $A$. calcoaceticus-baumannii complex was considered. The antimicrobial treatment was changed to cefepime after seven days of piperacillin/tazobactam and two days amikacin. After twelve days of antimicrobial treatment (including five days cefepime) the CT-scan showed persistent excavated lung lesions. Size of the largest cavity was slightly reduced and the walls were thinner.

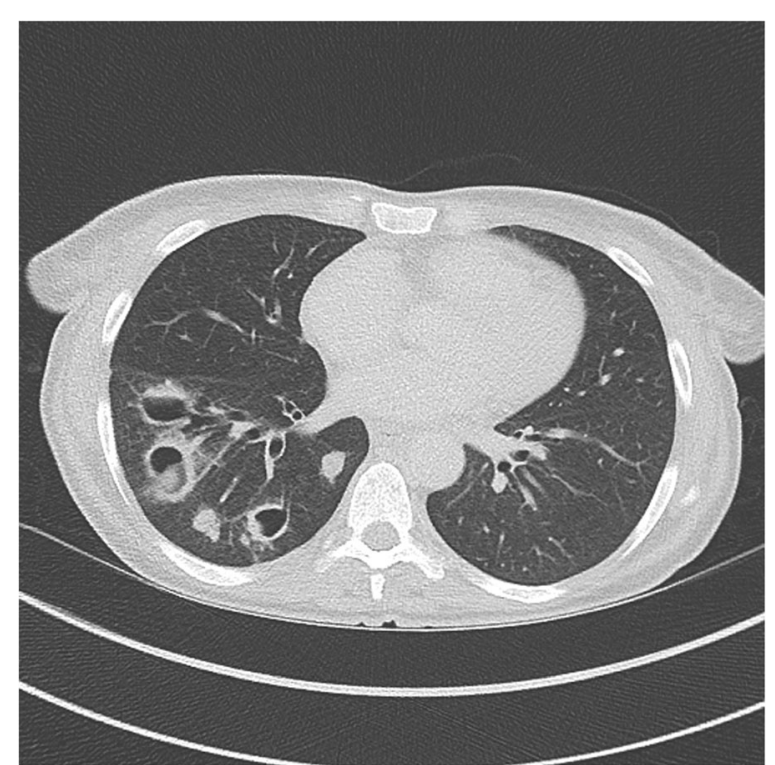

Fig. 3 Thoracic CT scan showing a cavitary community-acquired pneumonia due to $A$. pittii with the three others cavitations and nodules of the right lower lobe, three days after admission 
The clinical outcome was favourable after twenty-one days of antimicrobial treatment (including fourteen days cefepime). The Positon Emission Tomography - Computed Tomography performed at the end of the antimicrobial therapy did not find hypermetabolic fixation. Six months later, CT-scan showed residual excavated lung lesions of the right lower lobe. The bronchoscopy with biopsies was normal and the BAL cultures were negative.

\section{Species identification}

As species included in A. calcoaceticus-baumannii complex are closely related, rpoB sequencing and multilocus sequence typing (MLST) (bigsdb.web.pasteur.fr) [5] were performed to characterize the taxonomic status of the strain and the clone involved in this case. The isolate was identified as A. pittii belonging to ST249.

\section{Virulence study}

To evaluate the virulence of the $A$. pittii strain, we conducted a Caenorhabditis elegans nematode killing assay, a virulence model well described to study Acinetobacter spp. virulence [6]. The median time required to kill $50 \%$ of the Fer-15 C. elegans population (LT50) was compared between $A$. pittii isolate and an A. baumannii strain belonging to international clone II/ST2 isolated in our laboratory (NAB ST2). In vivo kinetics of killing of $C$. elegans infected by $A$. pittii and NAB ST2 were compared with the survival curve for worms fed on non-pathogenic E. coli (OP50) using a log-rank (Mantel-cox) test.

There was no significant difference between kinetics of killing of C. elegans infected by A. pittii ST249 and NAB ST2 compared with those fed on non-pathogenic E. coli
OP50, showing a very low virulence potential for the two strains equivalent to the standard feeding strain $E$. coli OP50 for nematodes (Fig. 4). The LT50 were $7 \pm 0.3$ days, $7 \pm 0.2$ days and $8 \pm 0.2$ days respectively for $A$. pittii ST249, NAB ST2 and E. coli OP50, $(p>0,05)$.

Given that biofilm formation is reported to favour bacterial colonization and persistence, biofilm-forming ability was investigated and kinetics of $A$. pittii ST249 and NAB ST2 were compared. The kinetics of the early phase of biofilm formation for A. pittii and NAB ST2 were studied using the BioFilm Ring Test ${ }^{\circ}$ (BioFilm Control, Saint Beauzire, France) [7]. A high BioFilm Index (BFI) value $(>8)$ corresponds to high mobility of free beads under magnetic attraction (no biofilm), while a low value $(\leq 2)$ corresponds to complete immobilisation of beads (strong biofilm) [8]. BFI values between 2 and 8 indicate that biofilm formation is in progress. Three experiments with two repeats were performed per strain and per incubation time. Mean BFI were compared between each strain after 1, 2, 3, 4 and $5 \mathrm{~h}$, using two-way Anova test followed by Bonferroni's multiple comparisons test. Statistics and graphs were prepared using the software package GraphPad Prism 6.0 ${ }^{\circ}$.

There were no significant differences between the two strains at the very beginning of kinetics $(\mathrm{BFI}=9.27 \pm 0.27$ for $A$. pittii and $8.63 \pm 0.37$ for NAB ST2 at $1 \mathrm{~h}$ of incubation, $p>0,05$ ) (Fig. 5). However, at the next time points, $A$. pittii ST249 showed a significantly slower biofilm formation than NAB ST2: BFI $=8.83 \pm 0.59$ vs $3.93 \pm 0.27$ respectively at $2 \mathrm{~h}(p<0.0001)$, $\mathrm{BFI}=6.3 \pm 0.17 \mathrm{vs} 1.87 \pm 0.12$ at $3 \mathrm{~h}(p<0.0001)$ and $\mathrm{BFI}=3.67 \pm 0.41$ vs $1.7 \pm 0.06$ after $4 \mathrm{~h}$ incubation

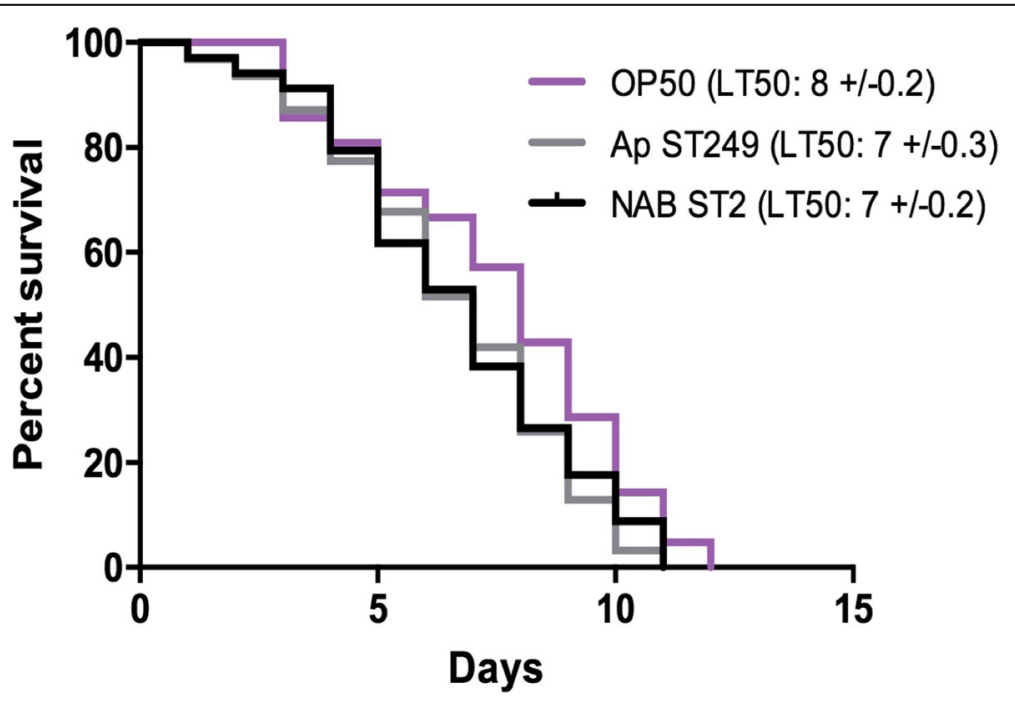

Fig. 4 In vivo kinetics of killing of C. elegans infected by A. pittii ST249 (Ap ST249) and A. baumannii ST2 (NAB ST2) compared with the survival curve for worms fed on non-pathogenic E. coli (OP50) using a log-rank (Mantel-cox) test to evaluate differences in survival rates between the different strains ( $p$ non-significant) 


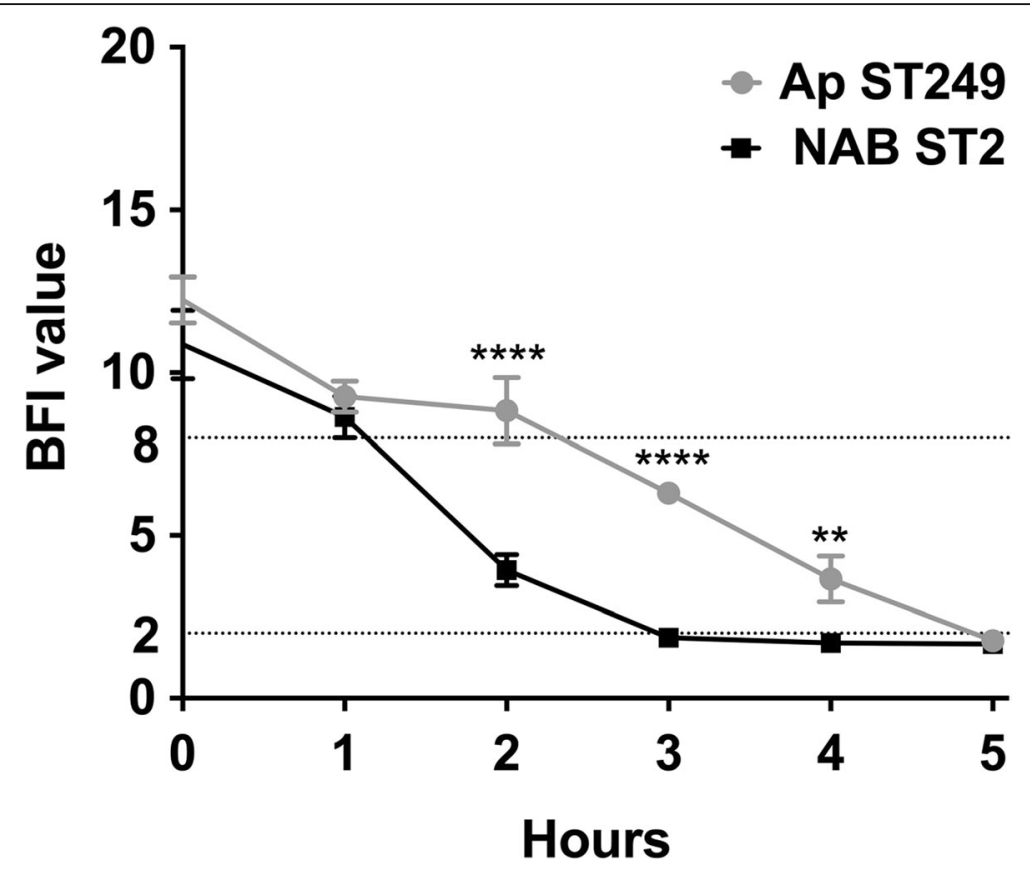

Fig. 5. Comparison between the kinetics of the early phase of biofilm formation for A. pittii ST249 (Ap ST249) and A. baumannii ST2 (NAB ST2). Means \pm standard deviations of BioFilm Indice (BFI) for at least three independent replicates are presented and significant differences between each strain at each time using two-way Anova test, followed by Bonferroni's multiple comparisons test are indicated by $*^{*}(p<0.01)$ and ${ }^{* * *}(p<0.0001)$

$(p<0.01)$. A. pittii biofilm was constituted after $5 \mathrm{~h}$ of incubation $(\mathrm{BFI}=1.77 \pm 0.09)$.

\section{Discussion and conclusions}

We report the first case of cavitary community-acquired pneumonia due to an $A$. pittii strain with low virulence potential, in a smoking patient with systemic lupus.

A. pittii is a member of the A. calcoaceticusbaumannii complex which also includes $A$. baumannii, A. calcoaceticus and A. nosocomialis. As a result of advances in molecular biology, A. pittii is increasingly recognized as a significant cause of nosocomial infection, particularly in intensive care unit setting, but nevertheless remains uncommon [3]. This bacterium is rarely observed to cause community-acquired infections notably cavitary pulmonary disease [1]. A. pittii ST249 has been described only twice, in two patients with hospitalacquired infection in Germany, a country where the prevalence of $A$. pittii seems high among species of $A$. calcoaceticus-baumannii complex [9]. The proportion of A. pittii community-acquired infections is not defined because of technological limitations in species identification across laboratories worldwide $[5,10]$. Prevalence may also be under-evaluated in healthy individuals because the low virulence profile does not result in clinical disease.

The large destruction of lung parenchyma with both cavitary lung lesions and pulmonary nodules in this case suggests that $A$. pittii is highly virulent. However, the $C$. elegans killing assays and the BioFilm Ring Test ${ }^{\circ}$ showed very low virulence potential and a poor ability to form biofilm as recently observed [11, 12]. The significant variability of virulence described among $A$. baumannii complex species probably explains the sub-acute clinical course unlike the fulminant evolution usually associated with Acinetobacter community-acquired pneumonia in tropical areas [12-14]. Moreover, the mortality rate for patients infected with $A$. pittii seems to be lower than for $A$. baumannii (15\% versus $40 \%)[4,10]$. Finally, the hosts' immune status and tobacco consumption seem to play a crucial role to facilitate infection. A. pittii and $A$. baumannii appear genetically and metabolically similar [15] and probably share the same risk factors for causing community-acquired infections: smoking, excessive alcohol consumption, diabetes mellitus and chronic lung disease $[13,14]$. In this case, the patient is diabetic, smokes and also has lupus, a condition known to increase the risk of infection with or without immunosuppressive drugs [16]. A. pittii is widely distributed in the environment and may contaminate food and animals, thus humans could acquire skin and/or oral carriage which subsequently favours infection [13, 17]. A study with more patients would allow us to better characterize the virulence of $A$. pittii and the host-pathogenic interaction but seems difficult due to the low prevalence of this type of infection.

With regard to drug choice, on the one hand, initial empiric antimicrobial therapy may be inappropriate if 
following standard practice guidelines for community acquired pneumonia, except if a fluoroquinolone is chosen [18]. On the other hand, second line treatment is ultimately most often appropriate because $A$. pittii is relatively susceptible to antibiotics used in this case $[4,6]$. The most effective drugs are carbapenems [5, 10]. Piperacillin/tazobactam, ticarcillin/clavulanate, ceftazidime or fluoroquinolone are alternative choices $[10,19]$. However, carbapenem resistance does occur frequently in the $A$. calcoaceticusbaumannii complex [5] and A. pittii ST249 has been described in association with the production of a carbapenemase GIM-1 [9]. In these cases, amikacin or colistin often remain the only therapeutic option.

In conclusion, one case of A. pittii communityacquired pneumonia revealed by cavitary lung lesions with a sub-acute clinical course lead to describing its low virulence profile. In the setting of immunocompromised patients and tobacco consumption, evaluation of $A$. pittii as a possible cause of community-acquired pneumonia should be considered, particularly in case of unsatisfactory response to first line antimicrobial therapy. The clinical course seems to be less severe than when caused by $A$. baumannii. As we can now more easily identify $A$. pittii in the A. calcoaceticus-baumannii complex, studying more patients could be interesting to asses the clinical features of this type of pneumonia.

\section{Abbreviations}

BAL: Broncho-alveolar lavage; BFI: BioFilm Indice; CFU: Colony Forming Unit; CT-scan: Computed tomography; EUCAST: European Committee on Antimicrobial Susceptibility Testing; LT50: Median time required to kill 50\% of the Fer-15 C. elegans population; MALDI-TOF: Matrix Assisted Laser Desorption lonisation/Time Of Flight; MLST: MultiLocus Sequence Typing; NAB ST2: A. baumannii strain belonging to international clone II/ST2; OP50: Non-pathogenic E. coli

\section{Acknowledgements}

Dr. Julie Carr, for reviewing this manuscript.

\section{Funding}

This research did not receive any specific grant from funding agencies in the public, commercial, or not-for-profit sectors.

\section{Availability of data and materials}

The datasets generated and analysed during the current study are not publicly available, but are stored in the medical record in Clinicom ${ }^{\circledR}$ software, in the Nimes University Hospital and are available from the corresponding author on reasonable request. Clinical data are reported according the CARE guidelines (http://www.care-statement.org/).

\section{Authors' contributions}

$\mathrm{RL}$ contributed to study design, literature search, data collection, analysis and interpretation, and writing. AP contributed to literature search, analysis and interpretation, and writing. EA contributed data collection and critical revision. JPL contributed to study design, literature search, data analysis and interpretation, writing and critical revision. AS contributed to study design, writing and critical revision. All authors read and approved the final manuscript.

Ethics approval and consent to participate Not applicable.

\section{Consent for publication}

Written consent for potentially identifying information being published as a case report was obtained from patient during admission. Consent was also reconfirmed verbally prior to submission of written article for publication.

\section{Competing interests}

None of the authors has any conflict of interest to declare that could influence this work.

\section{Publisher's Note}

Springer Nature remains neutral with regard to jurisdictional claims in published maps and institutional affiliations.

\section{Author details}

${ }^{1}$ Department of Internal Medicine, Caremeau University Hospital, 29 place du Professeur Debre, Nimes, France. ${ }^{2}$ Department of Microbiology, Caremeau University Hospital, 29 place du Professeur Debre, Nimes, France.

${ }^{3}$ Department of Infectious Diseases, Caremeau University Hospital, 29 place du Professeur Debre, Nimes, France.

Received: 6 April 2017 Accepted: 3 July 2017

Published online: 06 July 2017

References

1. Gadkowski LB, Stout JE. Cavitary pulmonary disease. Clin Microbiol Rev. 2008;21:305-33.

2. Lee Y-C, Huang Y-T, Tan C-K, Kuo Y-W, Liao C-H, Lee P-I, et al. Acinetobacter Baumannii and Acinetobacter genospecies $13 T \mathrm{TU}$ and 3 bacteraemia: comparison of clinical features, prognostic factors and outcomes. J Antimicrob Chemother. 2011;66:1839-46.

3. Nemec A, Krizova L, Maixnerova M, van der Reijden TJK, Deschaght $P$, Passet $\mathrm{V}$, et al. Genotypic and phenotypic characterization of the Acinetobacter calcoaceticus-Acinetobacter Baumannii Complex with the proposal of Acinetobacter pittii sp. nov. (formerly Acinetobacter genomic species 3 ) and Acinetobacter nosocomialis sp. nov. (formerly Acinetobacter genomic species 13TU). Res. Microbiol. 2011;162:393-404.

4. Chusri S, Chongsuvivatwong V, Rivera Jl, Silpapojakul K, Singkhamanan K, McNeil E, et al. Clinical outcomes of hospital-acquired infection with Acinetobacter nosocomialis and Acinetobacter pittii. Antimicrob Agents Chemother. 2014;58:4172-9.

5. De Vos D, Pirnay J-P, Biloca F, Jennes S, Verbeken G, Rose T, et al. Molecular epidemiology and clinical impact of Acinetobacter calcoaceticus-baumannii complex in a Belgian burn wound center. PLoS One. 2016;11:e0156237.

6. Vila-Farrés $X$, Ferrer-Navarro M, Callarisa AE, Martí S, Espinal P, Gupta S, et al. Loss of LPS is involved in the virulence and resistance to colistin of colistin-resistant Acinetobacter nosocomialis mutants selected in vitro. J Antimicrob Chemother. 2015;70:2981-6.

7. Chavant P, Gaillard-Martinie B, Talon R, Hébraud M, Bernardi T. A new device for rapid evaluation of biofilm formation potential by bacteria. Jicrobiol Methods. 2007:68:605-12.

8. Olivares E, Badel-Berchoux S, Provot C, Jaulhac B, Prévost G, Bernardi T, et al. The BioFilm ring test: a rapid method for routine analysis of Pseudomonas Aeruginosa biofilm formation kinetics. J Clin Microbiol. 2016;54:657-61.

9. Kaase M, Szabados F, Pfennigwerth N, Anders A, Geis G, Pranada AB, et al. Description of the metallo- $\beta$-lactamase GIM-1 in Acinetobacter pittii. J Antimicrob Chemother. 2014;69:81-4.

10. Wisplinghoff $H$, Paulus T, Lugenheim M, Stefanik D, Higgins PG, Edmond MB, et al. Nosocomial bloodstream infections due to Acinetobacter Baumannii, Acinetobacter pittii and Acinetobacter nosocomialis in the United States. J Inf Secur. 2012;64:282-90.

11. Lázaro-Díez M, Navascués-Lejarza T, Remuzgo-Martínez S, Navas J, Icardo JM, Acosta F, et al. Acinetobacter Baumannii and A. Pittii clinical isolates lack adherence and cytotoxicity to lung epithelial cells in vitro. Microbes Infect. 2016:18:559-64.

12. Na IY, Chung ES, Jung C-Y, Kim DH, Shin J, Kang K, et al. Comparison of the virulence-associated phenotypes of five species of Acinetobacter Baumannii Complex. J Microbiol Biotechnol. 2016;26:171-9.

13. Anstey NM, Currie BJ, Hassell M, Palmer D, Dwyer B, Seifert H. Communityacquired Bacteremic Acinetobacter pneumonia in tropical Australia is caused by diverse strains of Acinetobacter Baumannii, with carriage in the throat in at-risk groups. J Clin Microbiol. 2002;40:685-6. 
14. Antunes LCS, Visca P, Towner KJ. Acinetobacter Baumannii: evolution of a global pathogen. Pathog Dis. 2014;71:292-301.

15. Peleg AY, Seifert H, Paterson DL. Acinetobacter Baumannii: emergence of a successful pathogen. Clin Microbiol Rev. 2008;21:538-82.

16. Luijten RK Cuppen BV, Bijlsma JW, Derksen RH Serious infections in systemic lupus erythematosus with a focus on pneumococcal infections. Lupus 2014; 23:1512-1516.

17. Al Atrouni A, Joly-Guillou M-L, Hamze M, Kempf M. Reservoirs of nonbaumannii Acinetobacter species. Front Microbiol. 2016;7:49.

18. Mandell LA, Wunderink RG, Anzueto A, Bartlett JG, Campbell GD, Dean NC, et al. Infectious Diseases Society of America/American Thoracic Society consensus guidelines on the Management of Community-Acquired Pneumonia in adults. Clin Infect Dis. 2007:44:S27-72.

19. Burgess DS, Frei CR. Comparison of beta-lactam regimens for the treatment of gram-negative pulmonary infections in the intensive care unit based on pharmacokinetics/pharmacodynamics. J Antimicrob Chemother. 2005;56:893-8.

Submit your next manuscript to BioMed Central and we will help you at every step:

- We accept pre-submission inquiries

- Our selector tool helps you to find the most relevant journal

- We provide round the clock customer support

- Convenient online submission

- Thorough peer review

- Inclusion in PubMed and all major indexing services

- Maximum visibility for your research

Submit your manuscript at www.biomedcentral.com/submit 